\title{
Bericht über die gesamtpolnische Wissenschaftskonferenz: „In der Sorge um die Entwicklung der Hochschule" Bibliothek der Päpstlichen Universität Johannes Paul II. in Krakau, 28. November 2016
}

Am 28. November 2016 fand in der Bibliothek der Päpstlichen Universität Johannes Paul II. (UPJPII) die gesamtpolnische Konferenz „In der Sorge um die Entwicklung der Hochschule" statt. H. H. Prof. Dr. habil. Józef Stala, Prorektor der UPJPII und Hauptorganisator, eröffnete die Konferenz mit der Begrüßung der Referenten und der Gäste von Päpstlichen Universitäten aus dem ganzen Land: H. H. Prof. Dr. habil. Józef Bremer, Rektor der Ignatius-Akademie in Krakau, zusammen mit seinen Mitarbeitern; H. H. Prof. Dr. habil. Włodzimierz Wołyniec, Rektor der Päpstlichen Theologischen Fakultät in Breslau, auch mit seinen Mitarbeitern; H. H. Prof. Dr. habil. Krzysztof Pawlina, Rektor der Päpstlichen Theologischen Fakultät in Warschau, sowie alle weiteren Teilnehmer, insbesondere der Päpstlichen Universität Johannes Paul II. in Krakau und der Jagiellonen-Universität in Krakau.

Im ersten Referat „W kierunku rozwoju Uniwersytetu Papieskiego Jana Pawła II w Krakowie" (In welche Richtung sich die Päpstliche Universität Johannes Paul II. in Krakau weiterentwickelt) zeigte H. H. Prof. J. Stala ausgewählte Fakten der Universität auf - die Anzahl der Lehrstühle an der Universität (55\% selbstständige wissenschaftliche Mitarbeiter), die Anzahl der Professoren im Verhältnis zu den Studenten (11), den Anteil der im vergangenen Jahr erfolgreich finanzierten und realisierten Projekte (23\%). Im regionalen operativen 
Programm der Woiwodschaft Kleinpolen erhielt die Universität den Zuschlag für das Projekt „Sakralne Dziedzictwo Małopolski” (Das sakrale Erbe von Kleinpolen), das mit mehr als 4 Millionen Złoty finanziert wurde. Es wird ein internes Informationsbulletin herausgegeben, das auf aktuelle Angebote an Projekten und Stipendien hinweist. Darüber hinaus wurde die Zusammenarbeit mit der Ignatius-Akademie und der Polnisch-Amerikanischen Fulbright Kommission aufgenommen. Im Lauf der letzten beiden Jahre verdoppelte sich die Zahl der Studenten, Dozenten und Verwaltungsangestellten, die an der UPJPII registriert waren, auch konnte die Zahl der unterzeichneten bilateralen Vereinbarungen und Verträge um ein Drittel gesteigert werden. Der Referent verwies ebenso auf die Daten, welche die Aktivität einzelner Fakultäten belegen: die gemeinsame Organisation von zwei Wissenschaftskonferenzen (eine zusammen mit der Jagiellonen-Universität) sowie die von einer Vielzahl an Autoren in englischer Sprache publizierte Monographie „World Youth Days. A Testimony to the Hope of Young People", die gemeinsam mit der Jagiellonen-Universität vor dem Besuch von Papst Franziskus in Krakau veröffentlicht worden war. Die Fakultät für Gesellschaftswissenschaften hat zudem die Berechtigung erhalten, den Doktortitel für humanistische Studien im Bereich der Familienwissenschaften verleihen zu dürfen.

Prof. Dr. habil. Maciej Zabel, Vorsitzender des Evaluationskomitees für akademische Institutionen (KEJN), erinnerte in seinem Referat „Ewaluacja Jednostek Naukowych 2013-2016” (Evaluation der akademischen Institutionen von 2013 bis 2016) daran, dass die einzelnen Bewertungskategorien $A+, A, B$ und C mit dem Finanzierungsbedarf der satzungsgemäßen Tätigkeiten verknüpft wurden. Im Hinblick darauf, dass in Polen fast Tausend Institute an Universitäten und Hochschulen zu beurteilen sind, darunter etwa 750 Hochschulfakultäten, 76 Institute der Polnischen Wissenschaftsakademie (PAN), ungefähr 120 Forschungsinstitute und ca. 20 andere Einrichtungen, wird erkennbar, wie schwierig eine detaillierte Bewertung durchzuführen ist. Denn dies bedeutet für jede Beurteilung nahezu eine Million Einzelbetrachtungen, darunter mehr als 700000 wissenschaftliche Veröffentlichungen. Dieser Bewertungsprozess wird von lediglich 30 Spezialisten des Evaluationskomitees (KEJN) sowie weiteren 180 Experten und Mitarbeitern geleistet. Der Referent erläuterte vier grundlegende Kriterien für die Bewertung: 1. Die Errungenschaften in Forschung und Lehre; 2. Das Forschungspotenzial; 3. Die materiellen Effekte der Forschungsaktivitäten; 4. Weitere Effekte der Forschungsaktivitäten. In Bezug auf das erste Kriterium sind Publikationen, Monographien, Patente 
und künstlerische Aktivitäten von Bedeutung. Das zweite Kriterium erlaubt den Institutionen, ihre Berechtigungen zur Vergabe von akademischen Graden und Titeln, die Forschungsbasis sowie ihr Engagement bei Forschungsprojekten zu präsentieren. Dazu ist es erforderlich, wie der Professor betonte, nur auf die wichtigsten Errungenschaften zu verweisen und vernachlässigbare Informationen zu eliminieren. Das dritte Kriterium zeigt, welche Kosten von der jeweiligen Institution für die wissenschaftlichen Tätigkeiten, für Erwerbungen, Applikationen und Lehre aufgewendet werden. Das vierte Kriterium ermöglicht es den bewerteten Institutionen, weitere Errungenschaften aufzulisten, die in den vorangegangenen Kriterien nicht enthalten sind.

Im Anschluss daran trug Prof. Dr. habil. Stanisław Kistryn, Vorsitzender der Universitären Wissenschaftskommission und Prorektor der JagiellonenUniversität, sein Referat „Działalność statutowa Jednostek Naukowych” (Satzungen und Ordnungen der akademischen Institutionen) vor. Er verwies auf die veränderten Algorithmen bei der Zuteilung von satzungsgemäßen Dotierungen. So wurde die Zuteilung an die akademischen Institutionen aufgehoben und durch eine Basisdotierung ersetzt. Hinzu kam der Mittelwert N aus den zwei Folgejahren. Der Professor präsentierte die erneuerten statutengemäßen Dotierungen für akademische Institutionen in Abhängigkeit von ihrer speziellen Art und stellte die Dotierungen im Jahr 2015 und 2016 gegenüber, wie sie die zwanzig Universitäten betreffen, darunter auch die Päpstliche Universität Johannes Paul II. in Krakau. Er rechnete die Tendenz in der veränderten Dotierung auf eine Person an diesen Universitäten um, aber auch auf die einzelnen Fakultäten der Päpstlichen Universität Johannes Paul II. Er unterstrich eine deutliche wahrnehmbare Steigerung der Finanzierung pro Einzelperson an allen Fakultäten der UPJPII im Vergleichszeitraum der Jahre 2014 bis 2016. Zusammenfassend betonte der Referent, dass der angenommene Kostenbedarf im Bereich der Didaktik nicht adäquat im Hinblick auf die wissenschaftlichen Forschungen ist. Die Anzahl der akademischen Institutionen im System entspricht nicht der Forschungsinfrastruktur. Eine positive Folgerung stellt die Tendenz dar, dass die Durchschnittsdotierung auf eine gemeinsame, einheitliche Wertigkeit abzielt.

Dr. habil. Lucyna Rotter (Mitglied der Polnischen Akkreditierungskommission, 5. Kadenz) ging in ihrem Vortrag „Akredytacja Polskiej Komisji Akredytacyjnej - troska o jakość kształcenia" (Die Akkreditierung der Polnischen Akkreditierungskommission - in der Sorge um die Qualität der Bildung) auf aktuelle Akkreditierungskriterien ein, wie sie derzeit von der Polnischen 
Akkreditierungskommission angewendet werden. Dazu gehören: 1. Die Bildungskonzeption und deren Übereinstimmung mit der Mission sowie der Strategie der jeweiligen Hochschule; 2. Das Bildungsprogramm und die Möglichkeit, die erwarteten Bildungseffekte zu erzielen; 3. Die Effektivität des internen Systems, das die Qualität von Bildung und Lehre sicherstellt; 4. Ein Kader an geeigneten Mitarbeitern, die den Bildungsprozess durchführen; 5. Die Zusammenarbeit mit den örtlichen Behörden und der Wirtschaft im Rahmen des Bildungsprozesses; 6. Die internationale Ausrichtung des Bildungsprozesses; 7. Die Infrastruktur, die im Rahmen des Bildungsprozesses genutzt wird; 8. Die Betreuung der Studierenden sowie deren Unterstützung während ihres Lernprozesses sowie beim Erreichen der Bildungseffekte. Die Referentin unterstrich, dass das Hauptziel für das Bewertungsteam der Polnischen Akkreditierungskommission darin besteht, im Verlauf der Begutachtung die Bildungsqualität zu unterstützen, zu verstärken und zu erhöhen, nicht jedoch die Schwächen der jeweiligen akademischen Institution aufzuzeigen.

Dagegen verwies Dr. habil. Elżbieta Osewska (Mitglied der Polnischen Akkreditierungskommission, 4. Kadenz) in ihrem Referat „Weryfikacja efektów kształcenia i dobór odpowiednich metod kształcenia" (Die Verifikation von Bildungseffekten und die Auswahl geeigneter Bildungsmethoden) darauf, dass mit der Einführung der nationalen Qualifizierungsrahmen die Verantwortlichkeit der Hochschulen für die richtige Definition der Qualifikationen zugenommen hat. Dies muss sich dadurch bestätigen, dass der Absolvent alle geplanten Bildungseffekte erlangen kann. Lehre und Bildung müssen so organisiert sein, dass sie die Studenten in jeder Hinsicht unterstützen. Aus der Perspektive des Lehrenden werden nicht nur die grundlegenden Bildungseffekte von Bedeutung sein, sondern in gleicher Weise die Lehrmethoden und die Mechanismen der Verifikation. Für die Studierenden ist es wichtig, über Methoden zu verfügen, den eigenen Lernprozess zu verifizieren und Bildungsziele erreichen zu können. Die Referentin betonte, dass nicht die Ergebnisse der Examen für die Hochschulen am bedeutendsten sind. Vielmehr geht es darum, die Studierenden so gut vorzubereiten, dass sie die zukünftigen Prüfungen am Arbeitsplatz und in ihrem Lebensumfeld bestehen können. Obwohl die Mehrzahl der akademischen Institutionen die Bildungseffekte bewusst und korrekt definiert, treten dennoch weiterhin Probleme bei der Anwendung von Methoden für die Verifikation auf. Daher stellte sie die unterschiedlichen Methoden ausführlich vor - abhängig davon, welche Bildungseffekte sie nachprüfen können. Dabei verwies sie auch auf den jeweiligen Schwierigkeitsgrad und den Zeitbedarf. 
Insbesondere im Zusammenhang mit der in Kürze anstehenden Bewertung der Parameter für die Jahre 2013 bis 2016, traf die Konferenz auf großes Interesse der Teilnehmer, die eine Vielzahl an speziellen Fragen stellten. Sie unterstrichen vor allem deren Aktualität und Bedeutung sowie den Bedarf, weitere derartige Konferenzen zu organisieren.

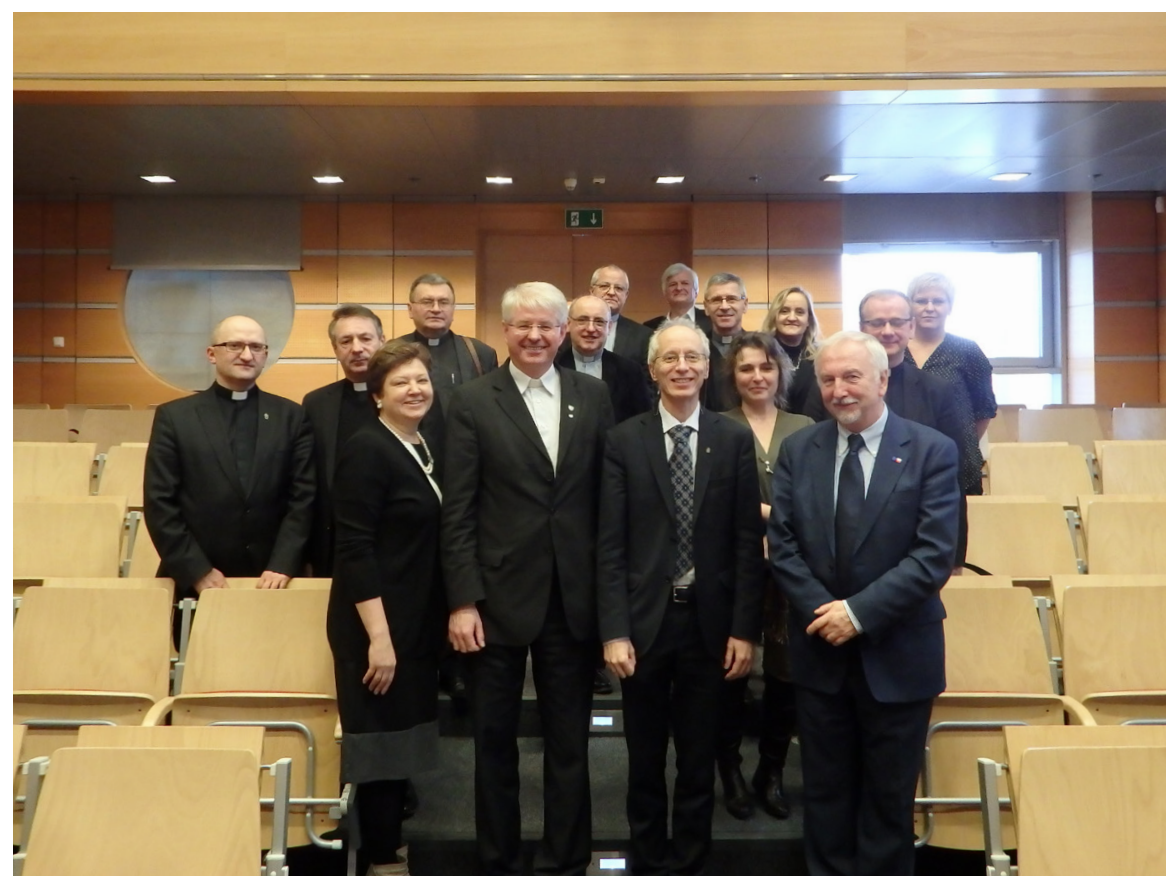

